\title{
NANOFLUID INJECTIVITY STUDY FOR ITS APPLICATION IN A PROCESS OF ENHANCED OIL RECOVERY (CEOR)
}

\author{
L. FERNÁNDEZ $Z^{\dagger}$ E. GONZÁLEZ \\ $\dagger$ Dto. de Química, Univ. Nac. del Comahue, 8300 Neuquén, Argentina \\ laura.fernandez@fain.uncoma.edu.ar \\ † Dto. de Geología y Petróleo, Univ. Nac. del Comahue, 8300 Neuquén, Argentina \\ esteban.gonzalez@fain.uncoma.edu.ar
}

\begin{abstract}
The application of tertiary recovery techniques through chemical injection (CEOR) is in full development in the mature oil fields of Argentina. An experimental study of nanofluids intended for enhanced oil recovery is presented in this work. A polyacrylamide solution prepared in brine with addition of silica nanoparticles was used as the focus of the study. Dynamic sweep tests of the displacement fluids in a laboratory-scale triaxial cell using a standard Berea sandstone cores that simulates the formation of the reservoir allow the calculation of parameters related to its injectivity, which take into account damage to the formation and blockade of poral throats , such as the resistance factor (FR), the residual resistance factor (FRR), the inaccessible pore volume (VPI) and the dynamic retention of the nanofluid (RD). The injection of the nanofluid carried out sequentially to the polymer solution has not produced an increase in the damage of the porous medium, so it is potential for its application in the displacement of crude oil.
\end{abstract} ing

Keywords - nanofluid, injectivity, chemical flood-

\section{INTRODUCTION}

The application of tertiary recovery techniques through chemical injection, CEOR, is in full development in the mature fields of Argentina. Attentive to this news, an interdisciplinary group of researchers from the University of Comahue -UNCo, develops a joint work of studies of chemical formulations and their applications in flood experiments in artificial core. After having reached record highs in the early 2000 `s, the production of hydrocarbons in Argentina has been in continuous decline for several years, unable to meet current needs and without ensuring a response to the increased future demand.

The primary and secondary recovery of oil achieves a combined efficiency of approximately $45 \%$, remaining an important residual oil in the reservoir. The fields are mature, with observed accumulations in classic entrapment systems and rocks with good petrophysical properties.

An increase in oil recovery in the immediate future depends on the success of the incipient enhanced recovery projects (Enhanced Oil Recovery-EOR) in mature reservoirs applying secondary. These processes consist in the injection of substances that are not originally present in the reservoir. Currently $40 \%$ of the oil is produced by secondary, but the wells are in their declining phase. The main companies have focused on the injection of special fluids such as polymers and their variants (sealing gels, microgels, Bright Water), alkalis, surfactants or a combination of them, a strategy known as enhanced recovery by chemical injection (Chemical Enhanced Oil Recovery-CEOR).

The injection of specifically designed polymer solutions (polyacrylamides-HPAM) is a modification of the water injection. It consists of adding a slug of polyacrylamides of high molecular weight prior to water injection into the reservoir, a strategy that allows improving the water-oil mobility ratio by increasing the viscosity of the injection fluid, resulting in a better displacement and a more complete sweep of the oil field with respect to the invasion with conventional water. Its selection is made in the screening stage and depends on reservoir conditions. The product must meet several requirements and the concentration to be used is specified to reach a target viscosity (Levitt and Pope, 2008).

With the recent surge of interest in polymer flooding, improved polymer products are manufactured, whose properties may be different from the similar polymers used during the earlier period of active chemical EOR applications

Several studies have shown that the addition of inorganic particles in nanometric dimensions (usually metal oxides, clays and carbides) can increase oil recovery, through different mechanisms, such as improving the rheological properties of polymer solutions (Ogolo et al., 2012); modifying water-oil interfacial tensions in systems with surfactants (Suleimanov et al., 2011) or having impact on the wettability of reservoir rock (Ogolo et al., 2012; Miranda et al., 2012; Mohsen et al., 2014).

To achieve the final objective of increasing oil recovery, preliminary displacing fluid studies (brine and / or polymer solution)-displaced fluid (crude) are required before proceeding to fluid-rock studies. However, the injectivity of the displacing fluid in the porous medium is crucial. This work focuses on the synthesis of a nanofluid and injectivity studies in dynamic flooding tests in a porous core for the determination of the parameters that determine its potential application, such as the resistance factor (FR) that accounts for the reduction of the mobility of the brine by the addition of the viscosifying agent, the residual resistance factor (FRR) that indicates the damage in the formation, and parameters associated with the blocking pore throats, such as the inaccessible pore volume (IPV) and dynamic retention (DR). To analyze the effect of the presence of nanoparticles, the injectivity of 
the nanofluid is compared with that of a polymer solution.

In this paper, through experiments in artificial cores, the influence of the nanofluid injection is discussed. The results are helpful for the further study of oil recovery.

\section{METHODS}

The composition of the brine selected for the nanoparticle size distribution study and for the preparation of the polymer solutions consists of the $\mathrm{NaCl}(6.2396 \mathrm{~g} / \mathrm{L})$, $\mathrm{CaCl}_{2} .2 \mathrm{H}_{2} \mathrm{O}(0.0889 \mathrm{~g} / \mathrm{L})$ and $\mathrm{MgCl}_{2} .6 \mathrm{H}_{2} \mathrm{O}(0.0715 \mathrm{~g} / \mathrm{L})$ which simulates a reservoir condition.

The polymer (P) selected was FP 3631S, a commercially hydrolyzed polyacrylamide (polyacrylate / polyacrylamide co-polymer) from the FLOPAAM тм polymer series, specially designed for CEOR applications, provided gently by SNF Floerger. The polymer is available as a dry powder, has a molecular weight of 20 million Daltons and an anionic charge between $25 \%$ and $30 \%$.

For the formulation of the nanofluid (NF), the size distribution (Dp) and the Zeta potential $(\xi)$ of various nanoparticles in brine and in a mixture of surfactants $(\mathrm{S})$ constituted by an Alcohol Alkoxy Sulfate and an branched Alkyl Benzene Sulfonate were investigated, on a NanozetaSizer-Malvern equipment. Nanoparticles of $\gamma$-alumina (544833 Sigma Aldrich) denoted Nano Al, nanoparticles of silica LUDOX AS-40 (Lot \# MKBK4442V, Aldrich), nanoparticles silica powder (DCP N150) denoted Nano $\mathrm{Si}$ and powdered silica microparticles (CemPLUS Geo D178) denoted Micro Si, were studied. For the preparation of the polymer and the nanofluid, the API RP 63 (1990) standard was followed. For the preparation of the nanofluid, $1 \mathrm{~g} / \mathrm{L}$ of nanoparticles was added to the stirring brine, allowing its dispersion, prior to the addition of the polymer powder. The polymer concentration was set at $1000 \mathrm{ppm}$.

The concentration of the initial polymer and in the effluent of the porous medium were measured by the chemical method of Bleach (API RP 63, 1990) and by rheology. The chemical method precipitates polyacrylamide from solution by adding acetic acid and bleach. The turbidity of the resulting suspension was measured with a colorimeter at $470 \mathrm{~nm}$ (UV-VIS Nanocolor -Macherey).

Considering the flow of the polymer solution from the well through the formation with a radial configuration, the injection rates in the injector and producer wells are relatively high, but decrease as the invasion proceeds and moves away from the well. Far away from the well, the polymer solution propagates at a low shear rate. For this reason it is necessary to know the rheological behavior in a range of shear rate, being 0.1-600 s-1 (Walker et al., 2012) the range corresponding to the flow in the typical reservoirs.

The rheological behavior of the polymer $(\mathrm{P})$ and the nanofluid (NF) was analyzed at $35^{\circ} \mathrm{C}$ with an Anton Paar MCR 302 Rheometer, using the geometry DG26.7 SN35071 in the rotational mode. Polyacrylamides have a non-Newtonian behavior, so that shear rates $(\gamma)$ were swept in the range $0.1-1000 \mathrm{~s}^{-1}$. In order to recreate the reservoir dynamic conditions the injection of three fluids, tracer (brine and tritium), polymer and nanofluid, flooding tests in artificial core were performed at $35^{\circ} \mathrm{C}$ according to API RP 63 (1990) in a standard porous core previously saturated with API1 brine (salinity $81 \mathrm{~g} / \mathrm{L}$ ). The experimental tests were performed in a triaxial cell at laboratory scale simulating the reservoir (Fig. 1).

As a porous medium for the laboratory coreflood, a standard Berea sandstone cores constituted mainly by quartz was selected, whose petrophysical characteristics were measured in our laboratory, Table 1.

The experiments described in this paper were all single-phase displacement of polymer and nanofluid solutions through consolidated sandstone. The core was prepared by evacuating and saturating with brine; the pore volume of the core was measured at this time. The experimental floods reported here were done in several consecutive stages:

1. Injection of $150 \mathrm{~mL}$ (approx $3 \mathrm{PV}$ ) of API1-quality water with tritium ( $4 \%$ ) added as a tracer.

2. Determination of the initial permeability to water (Kiw) by Darcy's law, assuming unidirectional and incompressible flow.

3. Injection of $150 \mathrm{~mL}$ of water API1 .

4. Determination of the concentration profile of Tritium as a tracer in the effluent (CT) by the liquid scintillation method.

5. Injection of $150 \mathrm{~mL}$ of brine as a tracer (API1 added with $10 \%$ of $\mathrm{NaCl}$ ).

6. Injection of $150 \mathrm{~mL}$ of water API1.

7. Determination of the concentration profile of brine as a tracer in the effluent (Cs) by the conductivity method.

8. Injection of $150 \mathrm{~mL}$ of polymer solution.

9. Determination of polymer permeability (Kp) by Darcy's law.

10. Injection of $150 \mathrm{~mL}$ of water API1.

11. Determination of polymer concentration profile in the effluent $(\mathrm{Cp})$ by the method of Bleach and rheology (API RP 63, 1990).

12. Determination of water permeability after the passage of polymer (Kpw).

13. Injection of $150 \mathrm{~mL}$ of nanofluid solution.

14. Determination of permeability to nanofluid (Knf) by Darcy's law.

15. Injection of $150 \mathrm{~mL}$ of water API1.

16. Determination of the concentration profile of nanofluid in the effluent (Cnf) by the method of Bleach and rheology (API RP 63, 1990).

17. Determination of the water permeability after the passage of the nanofluid (Knfw).

\begin{tabular}{|c|c|}
\hline Porosity $(\Phi)[\%]$ & 22.85 \\
\hline Water permeability $(\mathrm{kw})[\mathrm{mD}]$ & 90.00 \\
\hline Pore volume $(\mathrm{PV})\left[\mathrm{cm}^{3}\right]$ & 45.20 \\
\hline Apparent volume (VAP) $\left[\mathrm{cm}^{3}\right]$ & 197.87 \\
\hline Cross section $(\mathrm{A})\left[\mathrm{cm}^{2}\right]$ & 11.14 \\
\hline Length $(\mathrm{L})\left[\mathrm{cm}^{2}\right]$ & 17.76 \\
\hline Dry weight $(\mathrm{P})[\mathrm{g}]$ & 423.36 \\
\hline
\end{tabular}




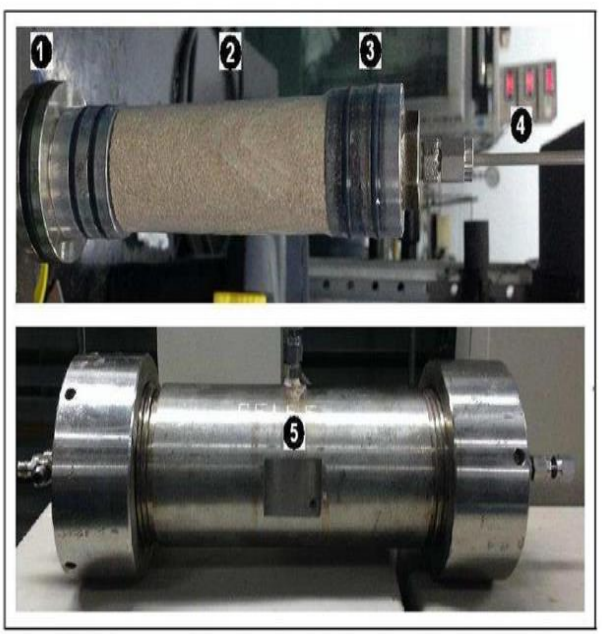

Figure 1: Triaxial Cell (holder) and Berea sandstone cores to the chemical flood experiments. (1) Fixed Top, (2) Berea core, (3) Mobile Top, (4) Internal pipe, (5) Holder cell and Packing rubber.

From the calculated permeabilities, the resistance factors (API RP 63, 1990) are evaluated after the polymer flooding, Eq. (1) and Eq. (2):

$$
\begin{gathered}
\text { FRp }=\frac{\lambda i w}{\lambda p}=\frac{\frac{K i w}{\mu w}}{\frac{K p}{\mu p}} \\
\text { FRRp }=\frac{\lambda i w}{\lambda p w}=\frac{\frac{K i w}{\mu w}}{\frac{K p w}{\mu w}}
\end{gathered}
$$

where $\lambda \mathrm{iw}(\mathrm{mD} / \mathrm{cP})$ is the initial brine rmobility, $\lambda \mathrm{p}$ $(\mathrm{mD} / \mathrm{cP})$ is the polymer mobility, $\lambda \mathrm{pw}(\mathrm{mD} / \mathrm{cP})$ is the brine mobility after the flow of the polymer, $\mu \mathrm{w}(1,11 \mathrm{cP})$ and $\mu \mathrm{p}\left(15 \mathrm{cP}\right.$ at $\left.\gamma=7.34 \mathrm{~s}^{-1}\right)$ are the viscosities of the brine and polymer respectively.

Analogously, the parameters after the flood with the nanofluid from Eq. (3) and Eq. (4) result:

$$
\begin{gathered}
\text { FRnf }=\frac{\lambda p w}{\lambda n f}=\frac{\frac{K p w}{\mu w}}{\frac{K n f}{\mu n f}} \\
\text { FRRnf }=\frac{\lambda p w}{\lambda n f w}=\frac{\frac{K p w}{\mu w}}{\frac{K n f w}{\mu w}}
\end{gathered}
$$

where $\lambda \mathrm{nf}(\mathrm{mD} / \mathrm{cP})$ is the nanofluid mobility, $\lambda \mathrm{nfw}$ $(\mathrm{mD} / \mathrm{cP})$ is the brine mobility after the flow of the nanofluid and $\mu \mathrm{nf}\left(17 \mathrm{cP}\right.$ at $\left.\gamma=7.34 \mathrm{~s}^{-1}\right)$ is the viscosity of the nanofluid.

When water-soluble, high molecular weight polymers are used for enhanced oil recovery (EOR), polymer retention (DR) retards propagation into the formation. The presence of the polymer is needed to provide high viscosity and low mobility levels - which in turn are needed to improve oil displacement and sweep efficiency. Consequently, high polymer retention can substantially delay oil displacement and recovery. Physical adsorption and mechanical entrapment are two major causes of polymer retention in porous media. To measure polymer retention (DR) in porous media the API RP 63 (1990) standard was followed. This method consists in the injection of a slug of polymer solution, followed by brine, and performing a mass balance on the polymer (i.e., retention $=$ polymer injected minus polymer produced).

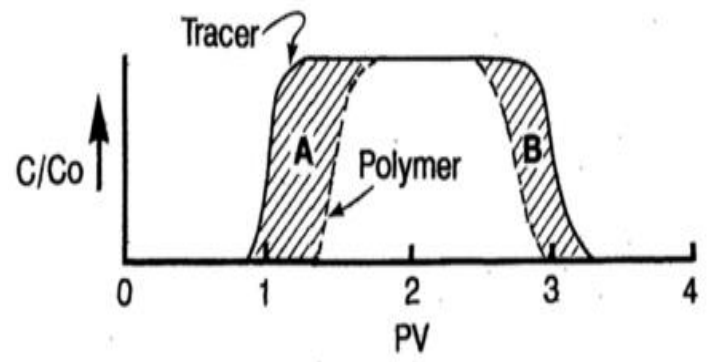

Figure 2: Polymer retention calculus and IPV (API RP 63, 1990).

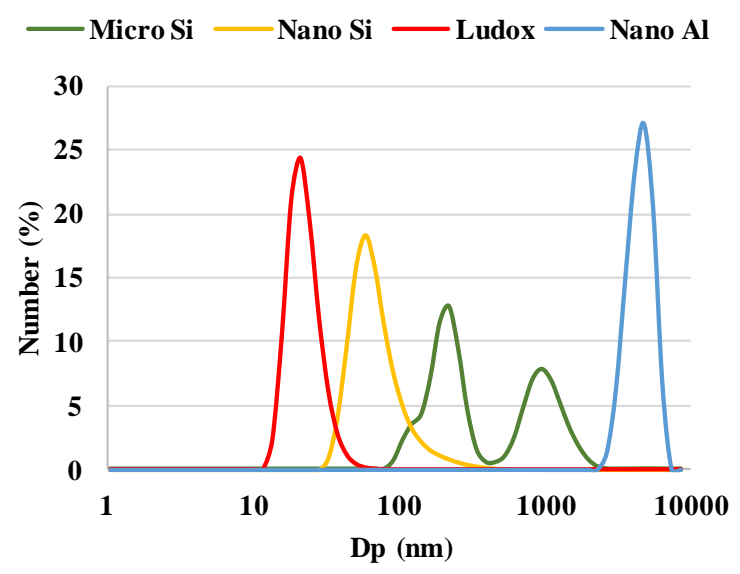

Figure 3: Size distribution of different nanoparticles dispersed in brine.

Dawson and Lantz (1972) have found that the solutions of typical water flooding polymers do not occupy all of the connected pore volume in porous media. The remainder of the pore volume is inaccessible to polymer (IPV). This inaccessible pore volume is occupied by water that contains no polymer. This allows changes in polymer concentration to be propagated through porous media more rapidly than similar changes in salt concentration. At the front edge of a polymer bank the effect of inaccessible pore volume opposite the effect of adsorption and may completely remove it in some cases.

Due to the existence of IPV, the polymer leaves the porous medium advanced with respect to the tracer. The IPV is calculated according to API RP 63 by area B of the Fig. 2.

It is important to note that IPV accelerates polymer propagation, whereas polymer retention (DR) retards it.

\section{RESULTS AND DISCUSSION}

Figure 3 presents the results of the size distributions of the different particles in brine (expressed in \% number). Figure 4 presents the result for those available in powder dispersed in a mixture of anionic surfactants (S). It can be noted that in brine the Ludox particles have a monomodal distribution centered at $20 \mathrm{~nm}$, the silica nanoparticles move at $78 \mathrm{~nm}$ and those of $\gamma$-alumina at $4598 \mathrm{~nm}$.

On the other hand, the microsilica particles have a bimodal distribution, centered at $206 \mathrm{~nm}$ and $1025 \mathrm{~nm}$. The $\gamma$-alumina particles are aggregated, because the $\mathrm{pH}$ of the solution $(\mathrm{pH}=8)$ is very close to its isoelectric point (Ta- 
ble 2). In all the analyzed cases, the presence of the surfactant allows to decrease the size of dispersed particles, at the expense of an increase in the Zeta potential (Table 2). The silica nanoparticles are displaced at $60 \mathrm{~nm}$, those of $\gamma$-alumina at $152 \mathrm{~nm}$, and the bimodal distribution of the microsilica at 22 and $105 \mathrm{~nm}$. For the preparation of the nanofluid, Ludox particles without surfactant are selected because they have the smallest dispersion size, taking into account that they must flow through the porous medium.

Rheological properties of the EOR polymer solution depend on the polymer properties and concentrations, salinity, shear rate and temperature (Lee et al., 2009). Polymer rheology in porous media affects both injectivity and sweep efficiency during a chemical flooding enhanced oil recovery (EOR) process. Figure 5 presents the rheological behavior of the displacing fluids.

At moderate to high fluid velocities in porous media (greater than $2 \mathrm{~s}^{-1}$ ), both polymer and nanofluid solutions show shear thinning that closely follows polymer's viscosity behavior (Lee et al., 2009). However, at low velocities in short cores (less than $2 \mathrm{~s}^{-1}$ ) the nanofluid continues to show shear thinning, such as the behavior reported for the xanthan (Seright et al., 2009), whereas viscosity data predicts Newtonian behavior for the polymer solutions. Because most oil is displaced at low velocities, this results are needed for accurate representation of nanofluid resistance factors when simulating chemical flooding processes.

Figure 5 presents the rheological behavior of the displacing fluids, demonstrating that the Ludox nanoparticles variably affect the behavior below shear rates of $2 \mathrm{~s}$ 1 , doubling the apparent viscosity $(\eta)$ for the lowest shear rate $\left(52 \mathrm{cP}\right.$ vs $26 \mathrm{cP}$ at $\left.0.1 \mathrm{~s}^{-1}\right)$. For greater deformations of the fluid, the particles are able to roll, without increasing the resistance to flow.

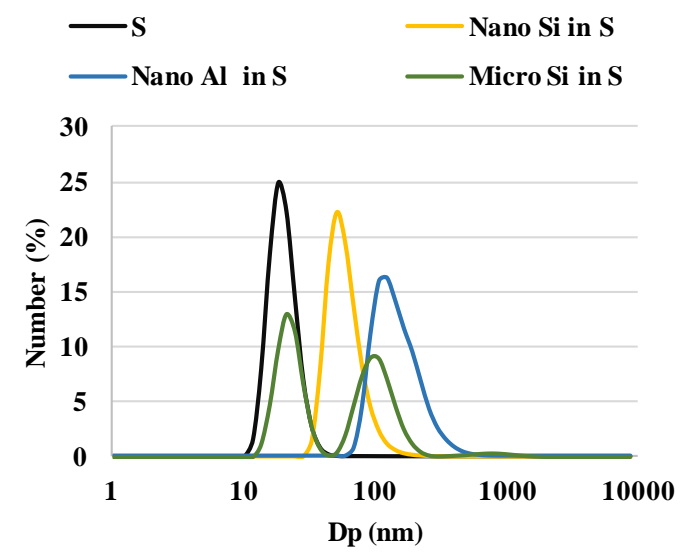

Figure 4: Size distribution of different nanoparticles dispersed in surfactant.

Table 2: Zeta Potencial results, $\xi(\mathrm{mV})$

\begin{tabular}{ccccc}
\hline \multicolumn{5}{c}{ Particles in Brine $\xi(\mathrm{mV})$} \\
\hline Ludox & MicroSi & NanoSi & NanoAl & $\mathrm{S}$ \\
$-27,5$ & $-32,1$ & $-27,8$ & $-2,3$ & - \\
\hline \multicolumn{5}{c}{ Particles in Surfactant $\xi(\mathrm{mV})$} \\
\hline- & $-42,5$ & $-60,6$ & $-62,1$ & $-61,3$ \\
\hline
\end{tabular}

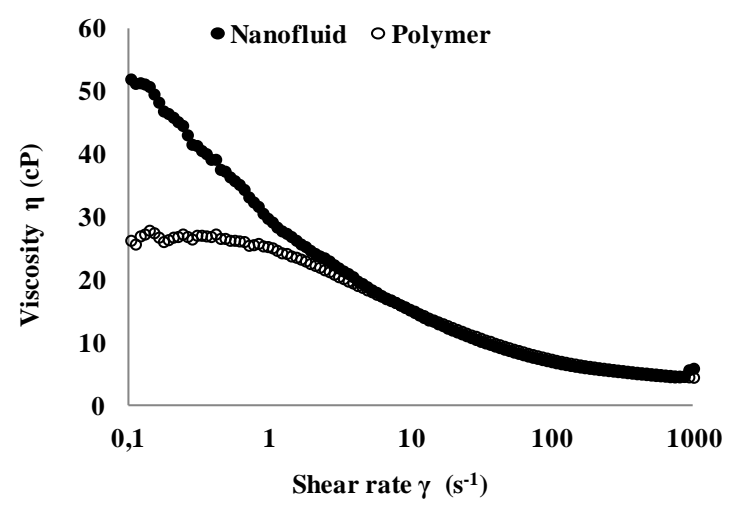

Figure 5: Rheological behavior of displacing fluids.

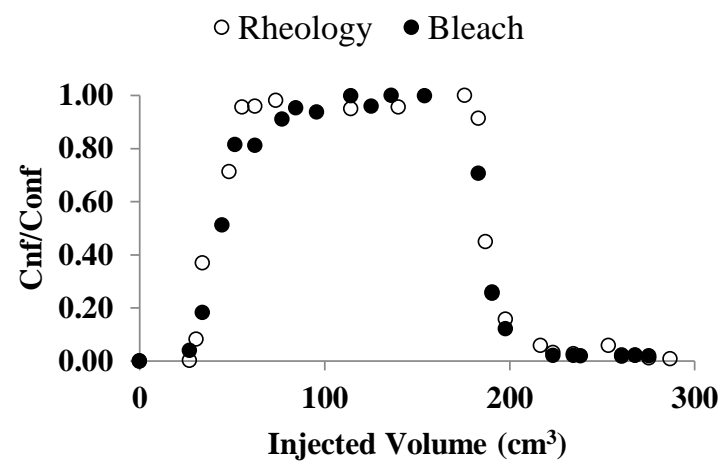

Figure 6: Concentration of the nanofluid by chemical bleach method and by rheology.

Dimensionless concentration profiles curves for the nanofluid from the flood are shown in Fig. 6 by the two measurement techniques demonstrating their agreement. The validation of the determination of the concentration by the rheological method is of great interest, since the chemical cannot be applied to all polyacrylamides, in the presence of hydrocarbons presents interference, is more expensive and destructive. Both methods are imprecise for concentrations below $15 \mathrm{ppm}$. The chemical method is calibrated for a concentration range between 20-250 ppm. Dilutions introduce errors in the measurement of concentrations. The rheological method contemplates the entire concentration range (up to $1000 \mathrm{ppm}$ ), so no dilutions are required. In addition, because it is non-destructive, the measurements of each sample can be repeated. Dimensionless concentration profiles curves for the polymer, tritium and brine from the flood are shown in Fig. 7, where C/Co corresponds to CT/CTo for tritium, Cs / Cso for the tracer brine, $\mathrm{Cp} / \mathrm{Cpo}$ for the polymer. Tritium is the best tracer and the one used in the field, but their quantification by liquid scintillation is much more expensive than brine. There is a good agreement in the profiles of the tracers, allowing to have alternative substances to carry out the chemical flooding studies.

The importance of a correct determination of the concentration profiles of the different fluids lies in the fact that they constitute the basis for the evaluation of the parameters that account for the fluid-rock interaction. These results are presented in Table 3. Resistance factor (FRp) is defined as brine mobility divided by polymer solution 
mobility. Assuming that the permeability of the core is fixed, resistance factor is the effective viscosity of the polymer solution in porous media relative to brine. When injecting fresh polymer solution at $10 \mathrm{ft} / \mathrm{d}$ resistance factors averaged was 82.88 , tripling the value corresponding to the zero-shear viscosity $(26 \mathrm{cP})$ for this solution. This finding was consistent with literature reports that HPAM solutions can provide somewhat higher effective viscosities (i.e., resistance factors) in porous media than in a viscometer (Seright et al., 2009) caused by polymer retention within the rock $(69.60 \mu \mathrm{g} / \mathrm{g})$. The retention also produces a residual damage in the porous medium represented by the FRRp, the which indicates a reduction in permeability close to half the initial value.

Figure 7 shows that the two fronts, which were injected simultaneously, have passed through the core with different velocities and emerged at different times. Moreover, the tracer pulse has emerged at the expected time after the injection of $1 \mathrm{PV}$. The polymer pulse emerged sooner; its velocity was greater than that of the tracer pulse. There is only one explanation: the pore volume through which the polymer can flow is smaller than the total pore volume of the core. The calculated average IPV was $12.6 \mathrm{~cm}^{3}$, there being a difference of $2.5 \%$ between both tracers (Table 3, polymer column).

Dimensionless concentration profiles curves for the nanofluid, tritium and brine from the flood are shown in Fig. 8.

For the nanofluid both tracers get the same value of IPV (17.60 $\mathrm{cm}^{3}$ ) (Table 3). However, given the existence of polymer within the porous medium, the increase of the IPV due to the flow of the nanofluid results of $11 \%$.With reference to the porous medium modified by the flow of the polymer, the nanofluid increases the remaining damage by $10 \%$ (FRRp) and increases the resistance factor by $50 \%$. Dynamic retention results from $51 \mu \mathrm{g} / \mathrm{g}$.

Zhang and Seright (2013) reports that: 1) HPAM polymer adsorption on a rock surface can be considered instantaneous and irreversible 2) If a porous medium is first contacted with dilute HPAM solution to satisfy the retention, no significant additional retention occurs when ex-

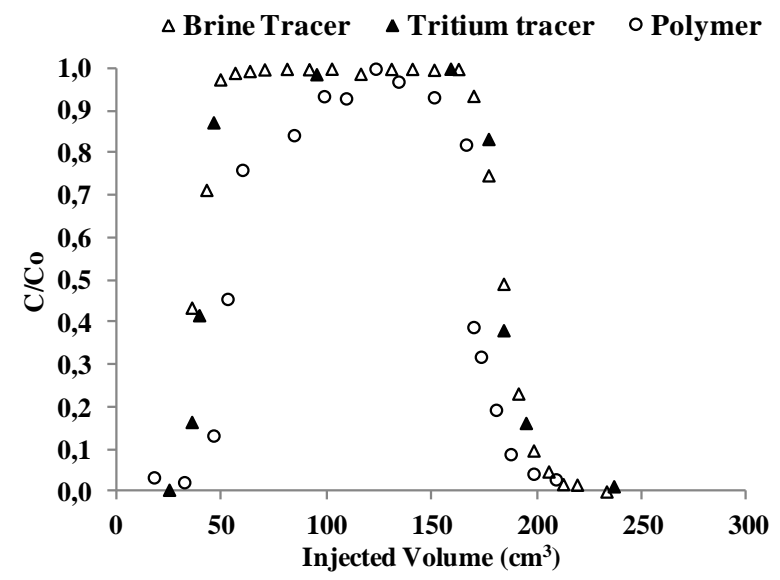

Figure 7: Concentration profiles in the effluent of the porous medium: Tritium, brine and polymer

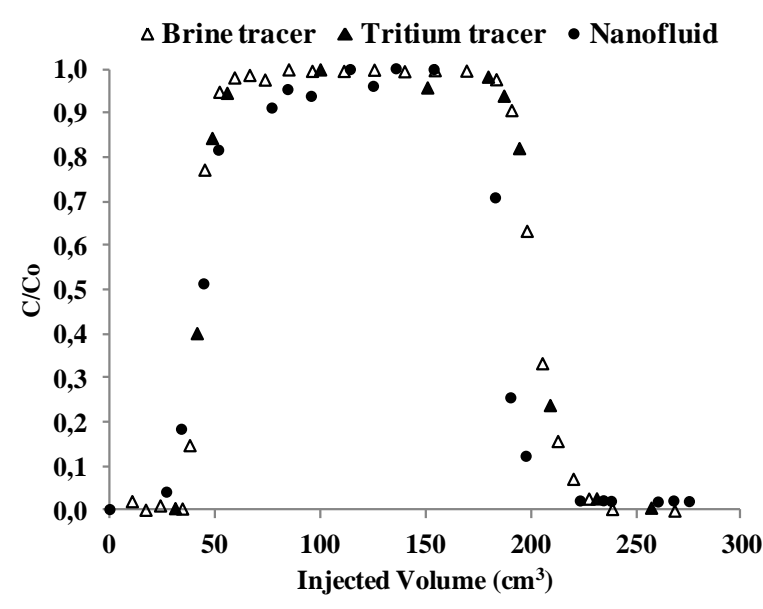

Figure 8: Concentration profiles in the effluent of the porous medium: Tritium, brine and nanofluid.

Table 3: Injectivity parameters

\begin{tabular}{cc}
\hline Polymer & Nanofluid \\
\hline FRp $=82.88$ & FRnf $=44.85$ \\
FRRp $=2.25$ & FRRnf $=1.10$ \\
Average IPV $\left(\mathrm{cm}^{3}\right)$ & Average IPV $\left(\mathrm{cm}^{3}\right)$ \\
12.60 & 17.60 \\
IPV Brine tracer & IPV Brine tracer \\
$28.30 \%$ & $39.10 \%$ \\
IPV Tritium tracer & IPV Tritium tracer \\
$25.80 \%$ & $38.90 \%$ \\
Average DR $(\mu \mathrm{g} / \mathrm{g})$ & Average DR $(\mu \mathrm{g} / \mathrm{g})$ \\
69.60 & 51.40 \\
DR Brine tracer & DR Brine tracer \\
70.00 & 52.80 \\
DR Tritium tracer & DR Tritium tracer \\
69.20 & 50.00 \\
\hline
\end{tabular}

posed to higher concentrations. In field applications ofpolymer and chemical floods, reduced polymer retention may be achieved by first injecting a low-concentration polymer bank.

Therefore the dynamic retention of the nanofluid can be attributed to the effect produced by the silica nanoparticles, which promote the interaction with the polymer inside the rock.

\section{CONCLUSIONS}

The two tracers used provide the information with a maximum difference of $2.5 \%$. Nanoparticles improved the rheological properties of polymer solution but increase the dynamic retention.

If the injection of nanofluid is carried out sequentially to the polymer, the injectivity parameters demonstrate its potential application in a process of CEOR.

Although it is potential for its application in the displacement of crude oil, a study to be carried out in the near future, it is important to emphasize that only injectivity does not guarantee an increase in recovery, since nanoscale particles-crude interphase phenomena that do not have been taken into account in this work. 


\section{REFERENCES}

API RP 63, Recommended Practices for Evaluation of Polymers Used in Enhanced Oil Recovery Operations, American Petroleum Institute (1990).

Dawson, R. and R. Lantz, "Inaccesible pore volume in polymer flooding," SPEJ, 12, 448-452 (1972).

Lee, S, D.H. Kim, C. Huh and G.A. Pope "Development of Comprehensive Rheological Property Database for EOR Polymers," SPE Annual Technical Conference and Exhibition, New Orleans, Louisiana, SPE 124798 (2009).

Levitt, D.B. and G.A. Pope, "Selection and Screening of Polymers in Enhanced Oil Recovery," SPE Symposium on Improved Oil Recovery, Tulsa, Oklahoma, USA, SPE 113845(2008).

Miranda, C.R., L.S. de Lara and B.C. Tonetto, "Stability and Mobility of Functionalized Silica Nanoparticles for Enhanced Oil Recovery Applications," SPE International Oilfield Nanotechnology Conference held in Noordwijk, The Netherlands, SPE 157033 (2012).

Mohsen, S.M., J. Moghadasi and S. Naseri, “An Experimental Investigation of Wettability Alteration in Carbonate Reservoir Using $\gamma$-Al2O3 Nanoparticles," Iranian Journal of Oil \& Gas Science and Technology, 3, 18-26 (2014).
Ogolo, N.A., O.A. Olafuyi and M.O. Onyekonwu, "Enhanced Oil Recovery using Nanoparticles," $S P E$ Saudi Arabia Section Technical Symposium and Exhibition, Al-Khobar, Saudi Arabia, SPE 160847 (2012).

Seright, R.S., M. Seheult and T. Talashek, "Injectivity Characteristics of EOR Polymers," SPE Res. Eval. \& Eng., 12, 783-792 (2009).

Suleimanov, B.A., F.S. Ismailov and E.F. Veliyev, "Nanofluid for enhanced oil recovery," Journal of Petroleum Science and Engineering, 78, 431-437 (2011).

Walker, D.L., C. Britton, D.H. Kim, S. Dufour, U. Weerasooriya and G.A. Pope, "The impact of Microemulsion Viscosity on Oil Recovery," Eighteenth SPE Improved Oil Recovery Symposium, Tulsa, Olklahoma, USA, SPE 154275 (2012).

Zhang, G. and R.S. Seright, "Effect of Concentration on HPAM Retention in Porous Media," SPE Annual Technical Conference and Exhibition, New Orleans, Louisiana, USA, SPE 166265 (2013).

Received February 10, 2018.

Sent to Guest Editor February 10, 2018.

Accepted December 28, 2018.

Recommended by Guest Editor Patricia M. Hoch 